\title{
The Usefulness of Laparoscopy in the Treatment of Incarcerated Internal Hernia through a Defect in the Broad Ligament
}

\author{
Sungwoo Cho, M.D., Sangchul Yun, M.D. \\ Department of Surgery, Soonchunhyang University Seoul Hospital, Seoul, Korea
}

\begin{abstract}
Hernias through the broad ligament are rare with a reported incidence of only 4-7\% of cases of internal hernias. Here, we report on a rare case of incarcerated internal hernia through a spontaneous defect in the broad ligament which was repaired using a laparoscopic technique. A 41-year-old female was admitted with colic abdominal pain. Laparoscopic exploration was performed for the diagnosis of intestinal obstruction. Laparoscopic examination confirmed an approximately 10-cm-long ileal loop herniated in the anterior to posterior direction through a left broad ligament defect. A large defect in the broad ligament was closed by an intracorporeal continuous running suture. The patient was highly satisfied with the cosmetic result. In conclusion, laparoscopy could be particularly helpful during pelvic surgery, which usually requires large skin incisions to ensure a good field of view. This approach was cosmetically effective, decreased the postoperative complications, and promoted early recovery to usual activity.
\end{abstract}

Keywords: Broad ligament, Hernia, Laparoscopy
Received June 24, 2015

Revised 1st August 21, 2015

2nd September 6, 2015

3rd September 14, 2015

Accepted September 15, 2015

Corresponding author

Sangchul Yun

Department of Surgery,

Soonchunhyang Vascular Center,

Soonchunhyang University Seoul

Hospital, Soonchunhyang University

College of Medicine, 59 Daesagwanro, Yongsan-gu, Seoul 04401, Korea Tel: +82-2-709-9240

Fax: +82-2-749-0449

E-mail: ultravascsurg@gmail.com

Copyright (c) 2015 The Journal of Minimally Invasive Surgery. All rights reserved.

This is an Open Access article distributed under the terms of the Creative Commons Attribution Non-Commercial License (http:/) creativecommons.org/licenses/by-nc/4.0/) which permits unrestricted non-commercial use, distribution, and reproduction in any medium, provided the original work is properly cited.

\section{INTRODUCTION}

The common causes of intestinal obstruction in adults include adhesions due to surgery, incarcerated inguinal hernias, malignant tumors, intussusceptions, inflammatory bowel disease, and volvulus. Internal hernias are rare but can cause small bowel obstruction. Paraduodenal hernia is a common type of internal hernia. ${ }^{1}$ Hernias through the broad ligament are very rare with a reported incidence of only 4 7\% of all cases of internal hernias. ${ }^{1,2}$ The disorder presents with various symptoms, but patients can be asymptomatic. Some patients complain of nausea, epigastric discomfort, and severe abdominal pain. However, delayed diagnosis can induce devastating conditions such as septic shock; therefore, the exact diagnosis and treatment are necessary. Here we report a rare case of incarcerated internal hernia through a defect in the broad ligament that was repaired using a laparoscopic technique after informed consent was provided.

\section{CASE REPORT}

A 41-year-old woman was admitted with colic abdominal pain accompanied by nausea that started 1 day prior. The patient had no history of abdominal surgery, traumatic delivery, or inflammatory bowel disease. A clinical examination revealed a distended and tympanic abdomen. Rectal examination results were normal. A pelvic examination revealed no cervical motion tenderness. Laboratory studies 

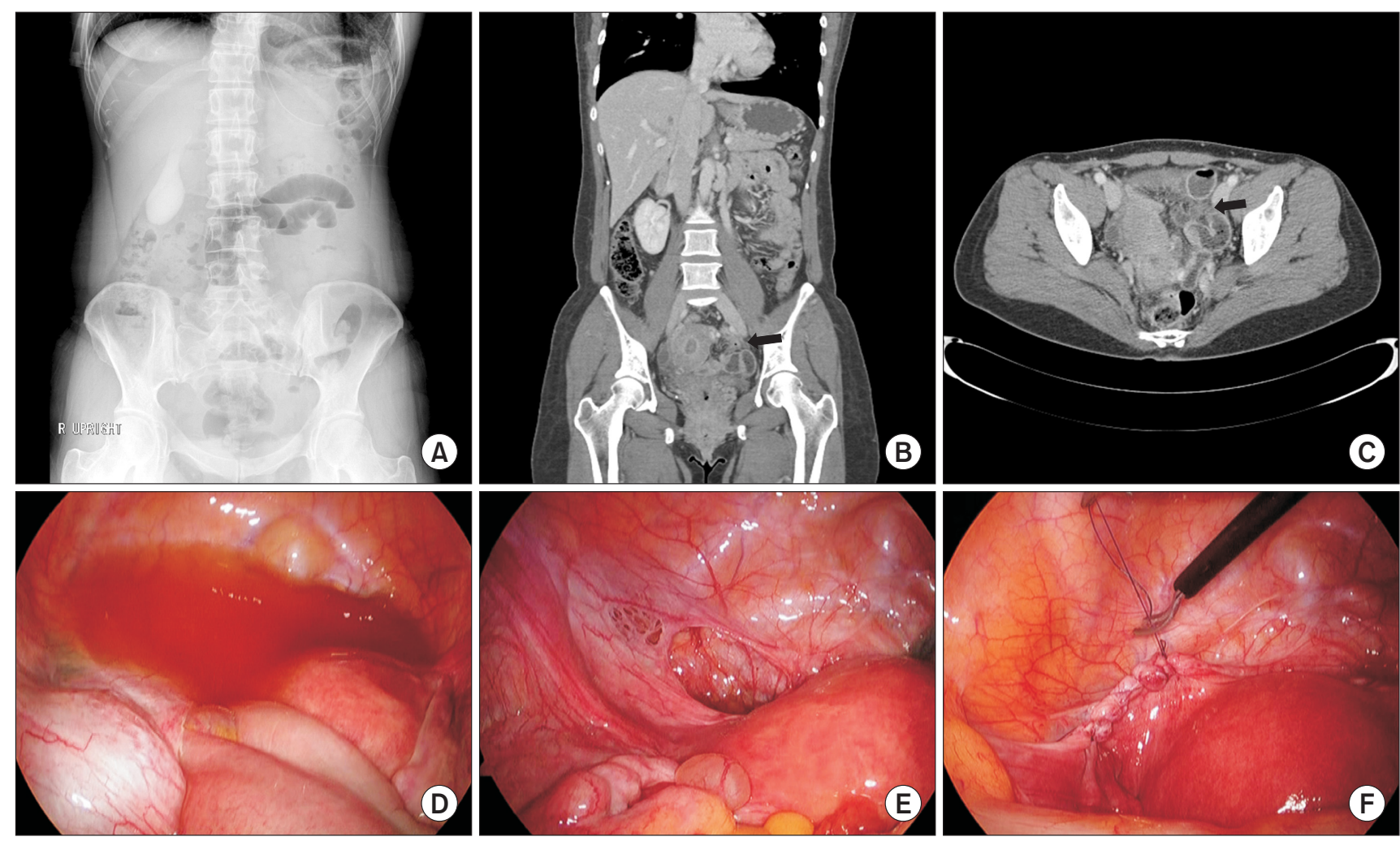

Fig. 1. (A) Simple abdominal computed tomography (CT) demonstrating high air-fluid levels in the small bowel. (B, C) Abdominopelvic CT: Short segmental enhancing wall thickening with mild luminal narrowing in the distal ileum and the pelvic ileal loop with angulation (black arrow). Fluid-filled dilatation of the ileal loop with evidence of a small amount of fecal matter in the bowel. (D) Intraoperative findings revealing ileal loop herniation through a large defect in the right broad ligament. (E) A huge defect at the left broad ligament. (F) The defect was closed to prevent recurrent herniation.

were normal except for a slightly elevated leukocyte count of $10.700 / \mathrm{mm}^{3}$. The patient's C-reactive protein level was 0.02 $\mathrm{mg} / \mathrm{dL}$. Plain abdominal computed tomography (CT) showed diffuse distended small bowel loops suggestive of an intestinal obstruction (Fig. 1A). Abdominal CT revealed short segmental enhancing wall thickening with mild luminal narrowing in the distal ileum and an angulated pelvic ileal loop. Fluid-filled dilatation of the ileal loop was noted with evidence of a small amount of fecal matter in the bowel consistent with a bowel obstruction (Figs. 1B, 1C). Although conservative treatment including Levin tube insertion, hydration, and antibiotic therapy were administered, the bowel passage did not improve.

Explorative laparoscopy was performed under general anesthesia. A 10-mm trocar was placed using a transumbilical skin incision. Two 5-mm trocars were placed at the right middle abdominal and suprainguinal areas (Fig. 2). Laparoscopic inspection confirmed an approximately $10-\mathrm{cm}$-long ileal loop herniated from the anterior to posterior side through a defect in the left broad ligament (Fig. 1D). The herniated small bowel loop seemed viable after laparoscopic reduction. A few pelvic ascites were cleared. A large defect in the broad

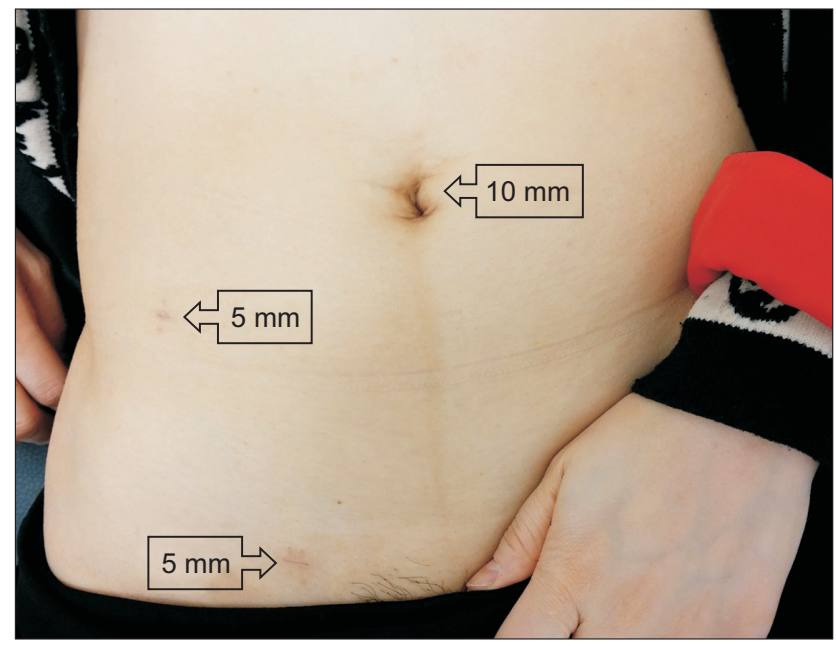

Fig. 2. A 10-mm trocar placed using a transumbilical skin incision. Two 5-mm trocars were placed at the right middle abdominal and suprainguinal areas. 
ligament was closed by an intracorporeal continuous running suture with Vicryl 3-0 to avoid recurrent herniation (Figs. 1E, $1 F)$. The patient was discharged on postoperative day 3 with no complications.

\section{DISCUSSION}

Cilley et al. ${ }^{3}$ classified broad ligament hernias by their anatomical location: type I defects, the most common type, occur through the entire wall of the broad ligament caudal to the round ligament of the uterus; type II defects occur above the round ligament through the mesosalpinx and mesovarium; and type III defects occur through the mesoligamentum teres. The patient in the present case had a type I fenestration defect, which involved complete fenestration through a defect in the broad ligament. This defect type occurs more commonly than the pouch type. ${ }^{2}$

Broad ligament defects are as likely to be congenital or acquired. Congenital defects are a consequence of spontaneous rupture of congenital cystic structures in the broad ligament reminiscent of the mesonephric or Müllerian ducts. ${ }^{4}$ This hypothesis could explain the incidence of the broad ligament defect in patients who are nulliparous or have never undergone pelvic surgery. ${ }^{5}$ Acquired defects may result from pelvis surgical trauma (such as Webster-Baldy operations), pregnancy, delivery, salpingitis, endometriosis, or pelvic inflammatory disease. ${ }^{6,7}$ Our patient's multiparity might have contributed to her development of the broad ligament defect.

The diagnosis is usually made perioperatively. Simple abdominal CT scans can reveal small bowel obstructions, but these findings are not specific for broad ligament herniation. Multi-detector row CT (MDCT) can be useful for making a preoperative diagnosis. ${ }^{8}$ The broad ligament can be identified because the $\mathrm{CT}$ depicts the round ligament, which is contained between the two leaves of the broad ligament. The diagnostic signs of this condition include mechanical obstruction with a double transition zone located in the pelvis. In the present case, CT showed a short segment of enhanced and thickened ileal wall with luminal narrowing and angulation in the pelvis.

Laparoscopy is an effective method in patients with internal obstruction. Based on a previous propensity score-matching process, laparoscopy showed a decreased rate of complications such as skin infections, sepsis, and the need for intraoperative transfusion. The use of laparoscopy also shortened the hospital stay. Furthermore, laparoscopy showed no significant difference in operative time, reoperation within 1 month, or mortality compared with open surgery. ${ }^{9}$

Despite the promising advances in laparoscopic treatment, no guidelines exist and there have been no randomized trials to date. A large study of 2005 patients with small bowel obstruction showed that $580(29 \%)$ underwent conversion to conventional laparotomy. Some factors are considered contraindications to laparoscopy surgery, including small bowel diameter $>4 \mathrm{~cm}$, documented history of dense adhesions, bowel ischemia, inadequate field of view, complex pathology such as malignancy, and inflammatory bowel disease. In contrast, an appendectomy history was thought to be associated with a higher chance of laparoscopic success. The number of previous operations is not considered a contraindication. ${ }^{10}$

In our case, the patient's condition did not improve with initial conservative treatment. However, her abdomen was not severely distended. During surgery, it was not difficult to place the trocars and obtain a good field of view. The patient was satisfied with the three minimal skin incisions made at the transumbilical, right suprainguinal, and right middle abdominal areas (Fig. 2). This approach was cosmetically effective, was painless, and featured early recovery to the patient's usual activities.

In conclusion, an internal hernia of the broad ligament is a rare cause of mechanical obstruction of the small intestine. CT can enable a preoperative diagnosis after a thorough analysis of signs and symptoms. Laparoscopy can be especially helpful during surgery for small bowel obstruction, which usually requires large skin incisions to ensure a good field of view. This approach was cosmetically effective, featured fewer postoperative complications, and enabled an early recovery to the patient's usual activities.

\section{REFERENCES}

1) Salar O, El-Sharkawy AM, Singh R, Speake W. Internal hernias: a brief review. Hernia 2013;17:373-377.

2) Haku T, Daidouji K, Kawamura H, Matsuzaki M. Internal herniation through a defect of the broad ligament of the uterus. Abdom Imaging 2004;29:161-163.

3) Cilley R, Poterack K, Lemmer J, Dafoe D. Defects of the broad ligament of the uterus. Am J Gastroenterol 1986;81:389-391.

4) Simstein NL. Internal herniation through a defect in the broad ligament. Am Surg 1987;53:258-259.

5) Ferrari S, Di Puppo F, Persico P, Ferrari A. Defects of the broad ligament of the uterus. J Minim Invasive Gynecol 2007;14:680681.

6) Redwine DB. Symptomatic internal hernia of the broad ligament: a complication of electrocoagulation therapy of endometriosis. Obstet Gynecol 1989;73:495-496.

7) Baron A. Defect in the broad ligament and its association with intestinal strangulation. Br J Surg 1948;36:91-94.

8) Quiroga S, Sarrias M, Sanchez JL, Rivero J. Small bowel obstruction secondary to internal hernia through a defect of the broad ligament: preoperative multi-detector CT diagnosis. Abdom 
Imaging 2012;37:1089-1091.

9) Lombardo S, Baum K, Filho JD, Nirula R. Should adhesive small bowel obstruction be managed laparoscopically? A National Surgical Quality Improvement Program propensity score analysis.
J Trauma Acute Care Surg 2014;76:696-703.

10) O'Connor DB, Winter DC. The role of laparoscopy in the management of acute small-bowel obstruction: a review of over 2,000 cases. Surg Endosc 2012;26:12-17. 